UNIVERSITY OF NOTTINGHAM

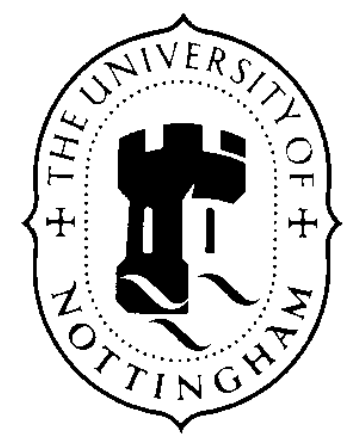

Discussion Papers in Economics

\title{
The welfare effects of entry: the role of the input market
}

By Arijit Mukherjee, Udo Broll \& Soma Mukherjee

December 2008 


\title{
The welfare effects of entry: the role of the input market ${ }^{*}$
}

\begin{abstract}
Arijit Mukherjee
University of Nottingham and The Leverhulme Centre for Research in Globalisation and Economic Policy, UK

Udo Broll

Dresden University of Technology, Germany

and

Soma Mukherjee

University of Nottingham, UK

December 2008

Abstract: In a successive Cournot oligopoly, we show the welfare effects of entry in the final goods market with no scale economies but with cost difference between the firms. If the input market is very concentrated, entry in the final goods market always increases welfare. If the input market is moderately concentrated, entry in the final goods market reduces welfare if the entrant is moderately cost inefficient than the incumbents. If the input market is highly competitive, entry in the final goods market reduces welfare if the entrant is very much cost inefficient than the incumbents. Hence, entry in the final goods market is more desirable under a concentrated input market. It follows from our analysis that entry increases the profits of the incumbent final goods producers if their marginal costs are sufficiently lower than the entrant's marginal cost.
\end{abstract}

Key Words: Entry; Profit; Vertical structure; Welfare

JEL Classification: L13; L50

Correspondence to: Arijit Mukherjee, School of Economics, University of Nottingham, University park, Nottingham, NG7 2RD, UK

E-mail: arijit.mukherjee@nottingham.ac.uk

Fax: +44-115-951 4159

\footnotetext{
* We would like to thank Michael Dobbins for helpful comments and suggestions. The authors are solely responsible for the views presented here but not the Universities. The usual disclaimer applies.
} 


\section{The welfare effects of entry: the role of the input market}

\section{Introduction}

The purpose of this paper is to consider the welfare effects of entry in the final goods market when the input market is imperfectly competitive and the entrant in the final goods market is cost inefficient than the incumbents. In this respect, we show that input-market concentration may play an important role.

The welfare effects of entry in imperfectly competitive markets have received attention for a long time. There are two strands of this literature. One strand of literature shows that entry in an imperfectly competitive market may be welfare reducing in the presence of scale economies. The earlier works in this area implicitly assume that the input markets are perfectly competitive, and therefore, entry does not affect the marginal costs of the firms (Williamson, 1968, Dixit and Stiglitz, 1977, von Weizsäcker, 1980, Perry, 1984, Mankiw and Whinston, 1986, Suzumura and Kiyono, 1987, Okuno-Fujiwara and Suzumura, 1993, Anderson et al., 1995 and Fudenberg and Tirole, 2000). Recent works look at the vertical structure ${ }^{1}$ where the marginal costs of the firms are endogenously determined (Ghosh and Morita, 2007a and b and Mukherjee, 2008). ${ }^{2}$ The second strand of the literature shows the welfare effects of

\footnotetext{
${ }^{1}$ It is needless to say that vertical relationship between the firms is quite common in real world. For example, automobile manufacturers purchase steel, tire and many other parts produced by other firms. The markets for microprocessors, aircraft-engines, packaged products and energy or power generating sectors are also characterized by vertical relationships. Komiya (1975) pointed out the industries such as iron and steel, petroleum refining, petrochemicals, cement, paper and pulp, and sugar refining with the tendency to develop excessive competition. While the industries mentioned in Komiya (1975) are characterized by homogeneous products and oligopoly, they produce intermediate goods for the final goods producers.

${ }^{2}$ In a recent paper, Mukherjee and Mukherjee (2008) show the welfare effects of entry in the presence of technology licensing, which affects the marginal cost of the licensee.
} 
entry in the absence of scale economies but under marginal cost asymmetries between the final goods producers. Klemperer (1988) shows that entry of a cost inefficient entrant may be welfare reducing. ${ }^{3}$ Ghosh and Saha (2007) confirm this conclusion with free entry. However, so far, this strand of the literature has ignored the role of the input markets. Our paper fills this gap by considering entry in a successive Cournot oligopoly where the entrant is cost inefficient than the incumbents. ${ }^{4}$

We show that if the input market is very concentrated, entry in the final goods market always increases welfare. If the input market is moderately concentrated, entry in the final goods market reduces welfare if the entrant is moderately cost inefficient than the incumbents. If the input market is highly competitive, entry in the final goods market reduces welfare if the entrant is very much cost inefficient than the incumbents. Hence, entry in the final goods market may be more desirable under a concentrated input market.

Given the input price, entry of a relatively cost inefficient entrant in the final goods market increases competition as well as creates production inefficiency by shifting output from the cost efficient incumbent final goods producers to the cot inefficient entrant. Hence, if the cost inefficiency of the entrant (compared to the incumbents) is sufficiently large, entry reduces welfare, as explained in the existing literature. However, entry in our analysis affects the input price due to the presence of an imperfectly competitive input market. We get that entry reduces the input price

\footnotetext{
${ }^{3}$ Lahiri and Ono (1988) show the welfare effects of cost reduction in a cost inefficient firm. Though they have not considered the issue of entry explicitly, a result similar to Klemperer (1988) follows from their analysis. Mukherjee (2007a) shows that entry increases welfare under Stackelberg competition irrespective of the marginal cost difference between the incumbent and the entrant.

${ }^{4}$ If horizontal merger is viewed as an opposite situation of entry, our paper may be related to the literature on the welfare effects of horizontal mergers in a vertical structure. See, e.g., Gans (2007) and the references therein.
} 
compared to no entry by increasing the elasticity of the input demand function, thus reducing the marginal costs of the incumbents. Since the input price effect depends on the input-market concentration, the welfare effects of entry in our analysis also depend on the input-market concentration.

It follows from our analysis that entry in the final goods market increases the profits of the incumbent final goods producers if the entrant is sufficiently cost inefficient than the incumbents. Tyagi (1999) and Naylor (2002) also show the profit raising effects of entry in a vertical structure. While Tyagi (1999) and Naylor (2002) respectively show the implications of the demand structure and the upstream agent's preference over the input price and input quantity, cost asymmetry between the incumbents and the entrant is responsible for our result.

The remainder of the paper is organized as follows. Section 2 describes the model and shows the results. Section 3 concludes.

\section{The model and the results}

Let us consider an economy with successive Cournot oligopoly as in Greenhut and Ohta (1976), Salinger (1988), Abiru et al. (1988), Ishikawa and Spencer (1999) and Ghosh and Morita (2007a), to name a few. Assume that there are $m \geq 1$ symmetric incumbent final goods producers and an entrant final goods producer denoted by firm $m+1$. As in Yoshida (2000), we assume that all final goods producers have Leontief technologies and use two inputs, say, input 1 and input 2 . Input 1 is produced in a perfectly competitive input market at a per-unit cost $d>0$. Hence, the price of this input is $d$. Input 2 is produced in an imperfectly competitive input market with $n \geq 1$ 
symmetric input suppliers, which produce like Cournot oligopolists and the corresponding input price is determined from the input demand function. Each of the $n$ input suppliers faces a constant marginal cost production, which is assumed to be zero for simplicity.

We assume that the entrant final goods producer is technologically inefficient than the incumbent final goods producers. There are several ways to model asymmetry between the final goods producers. We take a simple approach for analytical convenience. It is needless to say that our qualitative results are not sensitive to this modeling approach. We normalize each incumbent final goods producer's requirement for input 1 to zero, and assume that each incumbent final goods producer requires one unit of input 2 to produce one unit of the final good. However, the entrant final goods producer requires $\lambda$ units of input 1 and one unit of input 2 to produce one unit of the final good. Hence, denoting the price of input 2 by $w$, the marginal cost of each incumbent final goods producer is $w$, while the marginal cost of the entrant final goods producer is $w+c$, where $c=\lambda d$. Therefore, $c$ is the measure of cost inefficiency of the entrant compared to the incumbent final goods producers. Given $d$, as $\lambda$ reduces, it reduces the entrant's cost inefficiency compared to the incumbent final goods producers. ${ }^{5}$

Instead of considering two inputs, another way of considering asymmetry between the firms is to assume that all firms face the same input coefficients, but they differ in terms of other costs such as distribution costs. With this approach, the

\footnotetext{
${ }^{5}$ It is worth mentioning that different requirements for input 2 can also create cost asymmetries between the incumbent and the entrant final goods producers. However, our qualitative results remain under this alternative modeling strategy.
} 
distribution cost in our analysis is normalized to zero for the incumbent final goods producers and it is $c$ for the entrant.

Assume that the utility function of a representative consumer for the final good is

$$
U(q, I)=a q-\frac{q^{2}}{2}+H
$$

where $a>0, q$ is the total output of the final good and $H$ is a numeraire good. The utility function (1) gives the following inverse market demand function for the final good:

$$
P=a-q,
$$

where $P$ is price of the product.

We consider the following game. At stage 1, the profit-maximizing input suppliers produce their outputs like Cournot oligopolists. At stage 2, the profitmaximizing final goods producers choose their outputs like Cournot oligopolists and the profits are realized. We solve the game through backward induction.

In the following analysis, we will say that entry has occurred if the $m+1$ th firm is present in the final goods market. Therefore, under entry, there are $m+1$ firms producing in the final goods market. The marginal cost of the ith firm, $i=1,2, \ldots, m$, is $w$ and the marginal cost of the $m+1$ th firm is $w+c$. However, under no entry, the $m+1$ th firm does not produce in the final goods market. Therefore, under no entry, there are symmetric $m$ firms producing in the final goods market, and each of these firms face the marginal cost $w$. Hence, in the following analysis, the equilibrium values for the case of no entry are equivalent to the case of $c=0$ with $m$ as the total number of final goods producers. 


\subsection{The case of entry}

Let us start the analysis with entry. Hence, there are $m+1$ firms in the final goods market. Given the input prices, the $i$ th incumbent final goods producer and the entrant final goods producer (i.e., the $m+1$ th firm) maximize the following expressions:

$$
\begin{aligned}
& \underset{q_{i}}{\operatorname{Max}(a-q-w) q_{i}} \\
& \underset{q_{m+1}}{\operatorname{Max}}(a-q-w-c) q_{m+1},
\end{aligned}
$$

where $i=1, \ldots, m$ and $q=\sum_{1}^{n} q_{i}+q_{m+1}$.

The equilibrium output of each incumbent final goods producer and the equilibrium output of the entrant final goods producer are respectively

$$
q_{i}=\frac{a-w+c}{m+2} \quad \text { and } \quad q_{m+1}=\frac{a-w-c(m+1)}{m+2} .
$$

The total demand for input 2 is

$$
q=m q_{i}+q_{m+1}=I=\frac{a(m+1)-w(m+1)-c}{m+2} .
$$

The maximization problem for the $k$ th supplier of input 2 is, $k=1,2, \ldots, n$,

$$
\operatorname{Max}_{I_{k}} w I_{k}=\operatorname{Max}_{I_{k}} \frac{I_{k}(a(m+1)-I(m+2)-c)}{m+1},
$$

where $I=\sum_{1}^{n} I_{k}$. 
Given the symmetry of the suppliers of input 2, the equilibrium output of each supplier of input 2 is

$$
I_{k}=\frac{a(m+1)-c}{(n+1)(m+2)}, \quad k=1,2, \ldots, n .
$$

The total equilibrium supply of input 2 is

$$
I=n I_{k}=q=\frac{n(a(m+1)-c)}{(n+1)(m+2)}
$$

The equilibrium price of input 2 is

$$
w=\frac{a(m+1)-c}{(n+1)(m+1)}
$$

If $c>0$, the equilibrium price of input 2 reduces with the number of suppliers of input 2 but it increases with the number of final goods producers. Thus, we show that the number of final goods producers can affect the price charged by the imperfectly competitive input market in the presence of asymmetric cost final goods producers. This result modifies the “independence” result of Greenhut and Ohta (1976), where the input prices are independent of the number of symmetric cost final goods producers, and it is in line with Mukherjee (2007b) where the firms differ in terms of labor productivities and the input price depends on the number of final goods producers. The intuition for this result follows easily from Dhillon and Petrakis (2002), which show that the input prices are independent of the number of final goods producers if the equilibrium outputs and profits of the final goods producers are loglinear in the input price and the market features such as the number of final goods producers. ${ }^{6}$ It is immediate from (5) that the equilibrium outputs of the final goods

\footnotetext{
${ }^{6}$ The equilibrium output and profit of the $j$ th final goods producer is log-linear in the input price $z$ and the number of final goods producers $t$ if $q_{j}^{*}(z, t)=\psi_{j}(z) \phi_{j}(t)$ and $\pi_{j}^{*}(z, t)=\varphi_{j}(z) \vartheta_{j}(t)$.
} 
producers do not satisfy log-linearity in the input prices and the number of final goods producers.

Since the total final goods production is negatively related to the price of input 2 , it is worth noting that more suppliers of input 2 help to increase the total final goods production by reducing the price of input 2, while the higher price of input 2 due to more final goods producers partially offsets the positive effects of more final goods producers on the final goods production.

The profit of each incumbent final goods producer and the profit of the entrant final goods producer are respectively

$$
\begin{aligned}
& \pi_{i}=\frac{[\operatorname{an}(m+1)+c((n+1)(m+1)+1)]^{2}}{(n+1)^{2}(m+1)^{2}(m+2)^{2}}, \quad i=1,2, \ldots, m \\
& \pi_{m+1}=\frac{\left[\operatorname{an}(m+1)-c\left((n+1)(m+1)^{2}-1\right)\right]^{2}}{(n+1)^{2}(m+1)^{2}(m+2)^{2}} .
\end{aligned}
$$

Note that the entrant produces positive output if $c<\frac{a n(m+1)}{(n+1)(m+1)^{2}-1} \equiv c^{\max }$.

Welfare under entry, i.e., when the firm $m+1$ is present in the final goods market, is

$$
\begin{gathered}
W_{e}=U-c q_{m+1}=a q-\frac{q^{2}}{2}-c q_{m+1}+H \\
=\frac{a n[a(m+1)-c]}{(n+1)(m+2)}-\frac{n^{2}[a(m+1)-c]^{2}}{2(n+1)^{2}(m+2)^{2}}-\frac{c\left[a n(m+1)-c\left((n+1)(m+1)^{2}-1\right)\right]}{(n+1)(m+1)(m+2)}+H .
\end{gathered}
$$

\subsection{The case of no entry}


Now consider the case of no entry. If we put $c=0$ and consider the number of final goods producers as $m$, the equilibrium values shown in subsection 2.1 are equivalent to the case of no entry.

Under no entry, the equilibrium total supply of input 2 and the equilibrium price of input 2 are respectively

$$
I=n I_{k}=q=\frac{a n m}{(n+1)(m+1)}
$$

and $\quad w=\frac{a}{(n+1)}$.

The profit of each final goods producer is

$$
\pi_{i}=\frac{a^{2} n^{2}}{(n+1)^{2}(m+1)^{2}}, i=1,2, \ldots, m .
$$

Welfare under no entry is

$$
\begin{aligned}
W_{n e} & =U=a q-\frac{q^{2}}{2}+H \\
& =\frac{a^{2} n m}{(n+1)(m+1)}-\frac{a^{2} n^{2} m^{2}}{2(n+1)^{2}(m+1)^{2}}+H .
\end{aligned}
$$

\subsection{The effects of entry}

Now we are in position to see the effects of entry on the price of input 2, profits and welfare.

Proposition 1: If the entrant in the final goods market is cost inefficient than the incumbent final goods producers, the equilibrium price of input 2 is lower under entry than under no entry. 
Proof: Since $c>0$, the comparison of the equilibrium prices of input 2 shown in (10) and (15) proves the result. Q.E.D.

It is clear from (10) and (15) that if $c>0$, entry in the final goods market increases price elasticity of demand for input $2,{ }^{7}$ and helps to reduce the equilibrium input price.

Let us now compare the equilibrium profits of the incumbent final goods producers under entry and under no entry. The profit of each incumbent final goods producer is higher under entry than under no entry if

$$
\frac{[a n(m+1)+c((n+1)(m+1)+1)]^{2}}{(n+1)^{2}(m+1)^{2}(m+2)^{2}}>\frac{a^{2} n^{2}}{(n+1)^{2}(m+1)^{2}}
$$

or $\quad c>\frac{a n}{(n+1)(m+1)+1} \equiv c^{*}$,

where $c^{*}<c^{\max }$.

Hence, the following proposition is immediate.

Proposition 2: Entry in the final goods market increases the profit of each incumbent final goods producer if $c \in\left(c^{*}, c^{\max }\right)$.

\footnotetext{
7 The inverse demand functions for input 2 under entry and under no entry are respectively $w+\frac{c}{m+1}=a-\frac{q(m+2)}{m+1}$ and $w=a-\frac{q(m+1)}{m}$. Hence, if $c>0$, the price elasticity of demand for the former inverse demand function is $\frac{w}{a-w-\frac{c}{m+1}}$, which is higher than the price elasticity of
} demand for the latter inverse demand function, which is $\frac{w}{a-w}$. 
Entry in the final goods market has two effects on the profits of the incumbent final goods producers. First, for a given input price, entry in the final goods market reduces the profit of the incumbent final goods producers due to a higher competition in the final goods market. Second, entry in the final goods market reduces price of input 2, thus creating a positive effect on the profits of the final goods producers. If the entrant is sufficiently cost inefficient than the incumbents, i.e. $c$ is very high, the competition effect is negligible, while entry helps to reduce the marginal cost of the incumbent final goods producers due to the input price effect. In this situation, the outputs of the incumbent final goods producers are slightly lower under entry than under no entry, but they gain from an input price reduction on all inframarginal units. As a result, entry in the final goods market increases the profits of the incumbent final goods producers if $c$ is sufficiently high, i.e., $c \in\left(c^{*}, c^{\max }\right)$.

It is interesting to note that irrespective of the number of suppliers of input 2 and the number of final goods producers, there always exists $c$ such that entry in the final goods market increases the profits of the incumbent final goods producers. It is immediate from (16) that if the number of final goods producer increases (i.e., $m$ increases) it reduces $c^{*}$, but if the number of suppliers of input 2 increases (i.e., $n$ increases), it increases $c^{*}$. Therefore, initial higher competition in the final goods market increases the possibility of higher profit of the incumbent final goods producers due to entry in the final goods market. If competition in the final goods market is already very high, a further increase in competition due to entry does not have much effect on the profits of the incumbent firms, while entry creates a positive input price effect. In this situation, entry is more likely to increase the profits of the 
incumbent final goods producers. On the other hand, if the market for input 2 is already very competitive, which generates significantly lower input prices, entry in the final goods market does not have significant input price effect, while the negative competition effect tends to reduce the profits of the incumbent final goods producers.

Let us now see the welfare effects of entry in the final goods market. Given the equilibrium profits and the corresponding welfare under entry and under no entry, we cannot compare them generally. Hence, we use numerical examples to show our results. We subtract (17) from (13) and plot the difference in Figure 1 for $a=1$, $n=m=1$ and $c^{\max }=\frac{2}{7}$, in Figure 2 for $a=1, n=1, m=100$ and $c^{\max }=\frac{101}{20401}$, in Figure 3 for $a=1, n=20, m=100$ and $c^{\max }=\frac{2020}{214220}$, and in Figure 4 for $a=1$, $n=5, m=100$ and $c^{\max }=\frac{505}{61205} .^{8}$ Figure 1 corresponds to the case of entry in an existing monopolistic industry like Klemperer (1988). Figures 1 and 2 consider the situations where the input market is very much concentrated but the final goods market is concentrated in the former while sufficiently competitive in the latter. Figures 3 and 4 consider respectively the situations where the input market is very competitive and where the input market is moderately competitive.

\section{Figures 1, 2, 3 and 4}

In both Figures 1 and 2, welfare is higher under entry than under no entry for all feasible values of $c$. Hence, in contrast to Klemperer (1988), where entry in the final goods market reduces welfare for sufficiently large cost inefficiency of the entrant compared to the incumbent, we show that entry increases welfare for any feasible cost 
inefficiency of the entrant if the imperfectly competitive input market is very much concentrated. However, in Figure 3, where the imperfectly competitive input market is very competitive, the welfare effect of entry is similar to Klemperer (1988), i.e., entry reduces welfare if the entrant is sufficiently cost inefficient than the incumbents. Figure 4, which considers that the imperfectly competitive input market is moderately competitive, provides another interesting case where entry reduces welfare for moderate values of $c$, while it increases welfare for very high and for very low values of $c$. Though, given the complicated welfare expressions, we could not show our result generally, our examples show the importance of input market concentration, and suggest that the anti-competitive entry regulation may not be justified in a vertical structure if the input market is sufficiently concentrated. Even if the anticompetitive entry regulations may be justified if the input market is not very concentrated, the competitiveness of the input market plays an important role, and entry will be prevented for high values of $c$ if the input market is highly competitive, while it will be prevented for moderate values of $c$ if the input market is moderately competitive.

The above findings can be explained as follows. In our analysis, the welfare effect of entry in the final goods market can be decomposed into three separate effects. First, entry increases competition. Given the input price, higher competition in the final goods market tends to increase welfare. Second, entry shifts production from the incumbents to the entrant. Given the input price, the shift of production from the incumbents to the relatively cost inefficient entrant creates production inefficiency. This has a negative impact on welfare. These two effects are similar to

\footnotetext{
${ }^{8}$ We use 'The Mathematica 4' (see Wolfram, 1999) for the figures of this paper.
} 
the previous literature (Klemperer, 1988, Lahiri and Ono, 1988 and Ghosh and Saha, 2007). However, entry in our framework creates a third effect by reducing the price of input 2, which reduces the marginal cost of production in the final goods market and creates a positive effect on welfare.

If the input market is very much concentrated, the input price effect becomes significant. In this situation, the input price effect along with higher competition outweigh the negative effect of production inefficiency created by entry, thus creating a higher welfare under entry compared to no entry.

If the market for input 2 is very competitive, entry in the final goods market does not have a significant input price effect. Hence, the above-mentioned third effect is negligible, and entry in the final goods market reduces welfare if the entrant is sufficiently cost inefficient than the incumbent.

If the input market is moderately competitive, the input price effect is still an important factor and creates higher welfare under entry compared to no entry for the high values of $c$. However, as $c$ falls, it increases the input price, and reduces the benefit of the input price effect. Hence, the input price effect gets weaker for moderate values of $c$, and here entry reduces welfare. Though further reduction in $c$ makes the input price effect insignificant, it also reduces production inefficiency by making the entrant less cost inefficient. Therefore, for low values of $c$, entry again increases welfare by making the competition effect as the important factor.

Let us now see the reasons for the difference between our result and the previous works such as Klemperer (1988) and Lahiri and Ono (1988). If the input market is perfectly competitive, which can be the benchmark case of our analysis, the price of input 2 is zero (which is the cost of production for this input) irrespective of 
entry. Hence, standard calculation shows that, under perfectly competitive input market, entry occurs provided $c<\frac{a}{m+1} \equiv c_{\max }^{\text {bench }}$. In this benchmark case, welfare reducing entry occurs if $c<\frac{a(3+2 m)}{3+9 m+8 m^{2}+2 m^{3}} \equiv \widehat{c} \cdot{ }^{9}$ Recall that the reason for welfare reducing entry is due to the creation of production inefficiency in the industry by shifting output from the cost efficient incumbent to the cost inefficient entrant. If the entrant is very cost inefficient, the production inefficiency effect dominates the competition effect, thus reducing welfare.

Since highly competitive input market in our analysis approximates a perfectly competitive input market, we get a result similar to Klemperer (1988) if the market for input 2 is very much competitive. However, if the market for input 2 is not very competitive, the input price effect plays an important role in determining the welfare effects of entry.

The first effect that we observe in a vertical structure is that entry in the vertical structure occurs for a small range of $c$, since $c^{\max }<c_{\max }^{\text {bench }}$. Therefore, by making entry unprofitable for very high values of $c$, the vertical structure eliminates the possibility of welfare reducing entry for very high values of $c$.

Since $\widehat{c}<c^{\max }$, entry in the vertical structure still occurs for those high values of $c$ for which entry reduces welfare in the absence of vertical structure. However, if $c$ is very high, it follows from Proposition 2 that entry increases profits of the incumbent final goods producers. Hence, for very high values of $c$, entry in our

\footnotetext{
9 In the benchmark model, welfare under entry and no entry are given by $W_{e}^{\text {bench }}=\frac{2 m(a+c)^{2}+2(a-(m+1) c)^{2}+(a(m+1)-c)^{2}}{2(m+2)^{2}}$ and $W_{n e}^{\text {bench }}=\frac{a^{2} m(m+2)}{2(m+1)^{2}}$.
} 
analysis actually creates production efficiency by reducing the input price (see Proposition 1), thus increasing the outputs of the incumbent final goods producers.

However, entry reduces the outputs of the incumbent final goods producers if $c<c^{*}$, where $c^{*}$ is generally higher than $\widehat{c}$. Hence, there still remains a range of $c$, which is $\left[\widehat{c}, c^{*}\right]$, over which entry in the vertical structure reduces profits of the incumbent final goods producers and it reduces welfare in the absence of a vertical structure. In this situation, though entry reduces the outputs and the profits of the incumbent final goods producers and creates production inefficiency, the positive input price effect may increase welfare as explained above.

As a final remark, we have focused on the cost inefficiency of the entrant compared to the incumbent final goods producers. However, if the entrant is cost efficient than the incumbents, entry does not create the above-mentioned production inefficiency. Hence, welfare in our framework will always increase if the entrant is cost efficient than the incumbents.

\section{Conclusion}

Several authors have analyzed the welfare effects of entry in oligopolistic markets. However, vertical relationship did not receive due attention in the literature, though several industries are characterized by vertical relationships. In a successive Cournot oligopoly that explicitly incorporates vertical relationship, we show the welfare effects of entry in the final goods market.

Our results suggest that entry in the final goods market is more desirable if the input market is concentrated. In this situation, entry creates a significant positive 
benefit by reducing the input price, which, in turn, helps to reduce the marginal costs of final goods production. We also show that entry in the final goods market increases the profits of the incumbent final goods producers if they are sufficiently cost efficient than the entrant.

We show the implications of cost asymmetries in the presence of vertical relationships and no scale economies. However, following Ghosh and Morita (2007a), we conjecture that if there are free entry and scale economies, the possibility of higher welfare under entry in our analysis may either increase or decrease. More entrants tend to increase welfare, while the entry cost tends to reduce welfare. The net effect depends on the relative strengths of these factors. In general, the industrial structure that differs in terms of vertical relationship, the cost asymmetry between the firms and scale economies play important roles in determining the welfare effects of entry. 


\section{References}

Abiru, M., B. Nahata, S. Raychaudhuri and M. Waterson, 1998, 'Equilibrium structures in vertical oligopoly', Journal of Economic Behavior and Organization, 37: 463-80.

Anderson, S. P., A. de Palma and Y. Nesterov, 1995, 'Oligopolistic competition and the optimal provision of products', Econometrica, 63: 1281-1301.

Dhillon, A. and E. Petrakis, 2002, 'A generalised wage rigidity result', International Journal of Industrial Organization, 20: 285-311.

Dixit, A. and J. Stiglitz, 1977, 'Monopolistic competition and optimum product diversity', American Economic Review, 67: 297-308.

Fudenberg, D. and J. Tirole, 2000, 'Pricing a network good to deter entry', The Journal of Industrial Economics, XLVIII: 373-90.

Gans, J., 2007, 'Concentration-based merger tests and vertical market structure', Journal of Law and Economics, 50: 661-80.

Greenhut, M. L. and H. Ohta, 1976, 'Related market conditions and interindustrial mergers', The American Economic Review, 66: 267-77.

Ghosh, A. and A. Morita, 2007a, 'Free entry and social efficiency under vertical oligopoly’, Rand Journal of Economics, 38:539-52.

Ghosh, A. and A. Morita, 2007b, 'Social desirability of free entry: a bilateral oligopoly analysis’, International Journal of Industrial Organization, 25: 925-34.

Ghosh, A. and S. Saha, 2007, 'Excess entry in the absence of scale economies', Economic Theory, 30: 575-86. 
Ishikawa, J. and B. J. Spencer, 1999, 'Rent-shifting export subsidies with an imported intermediate product', Journal of International Economics, 48: 199-232.

Klemperer, P., 1988, 'Welfare effects of entry into markets with switching costs', The Journal of Industrial Economics, XXXVII: 159-65.

Komiya, R., 1975, 'Planning in Japan', in M. Bornstein (Ed.), Economic planning: east and west, Ballinger: Cambridge, MA.

Mankiw, A. G. and M. D. Whinston, 1986, 'Free entry and social inefficiency', RAND Journal of Economics, 17: 48-58.

Mukherjee, A., 2007a, 'Entry in a Stackelberg perfect equilibrium', Economics Bulletin, 12 (33): 1-6.

Mukherjee, A., 2007b, 'Note on a generalized wage rigidity result', Economics Bulletin, 10 (12): 1-9.

Mukherjee, A., 2008, 'Excessive entry in a bilateral oligopoly’, Discussion Paper, 08/02, School of Economics, University of Nottingham.

Mukherjee, A. and S. Mukherjee, 2008, 'Excess-entry theorem: the implications of licensing', The Manchester School, 76: 675-89.

Naylor, R. A., 2002, ‘The effects of entry in bilateral oligopoly’, Mimeo, University of Warwick.

Okuno-Fujiwara, M. and K. Suzumura, 1993, 'Symmetric Cournot oligopoly and economic welfare: a synthesis', Economic Theory, 3: 43-59.

Perry, M. K., 1984, ‘Scale economies, imperfect competition, and public policy’, The Journal of Industrial Economics, 32: 313-33.

Salinger, M. A., 1988, 'Vertical mergers and market foreclosure', The Quarterly Journal of Economics, 103: 345-56. 
Spence, A.M., 1976, 'Product selection, fixed costs, and monopolistic competition', Review of Economic Studies, 43: 217-36.

Suzumura, K. and K. Kiyono, 1987, 'Entry barriers and economic welfare', The Review of Economic Studies, 54: 157-67.

Tyagi, R. K., 1999, 'On the effects of downstream entry', Management Science, 45: 59-73.

von Weizsäcker, C. C., 1980, ‘A welfare analysis of barriers to entry’, Bell Journal of Economics, 11: 399-420.

Williamson, O., 1968, 'Economies as an antitrust defense: welfare trade-offs', American Economic Review, 58: 18-36.

Wolfram, S., 1999, The mathematica book, $4^{\text {th }}$ Ed., Wolfram Media, Cambridge University Press. 


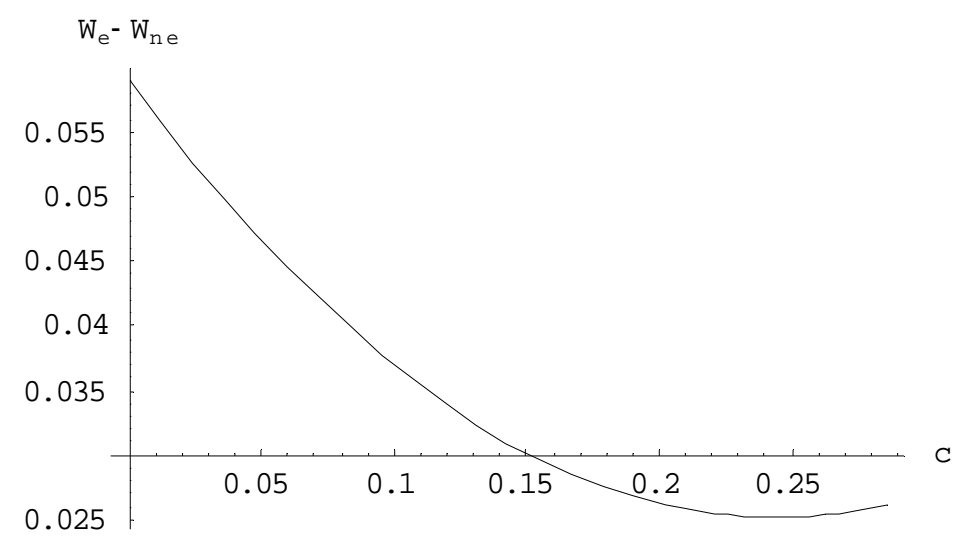

Figure 1: Subtracting (17) from (13) for $a=1, n=m=1$ and $c^{\max }=\frac{2}{7}$.

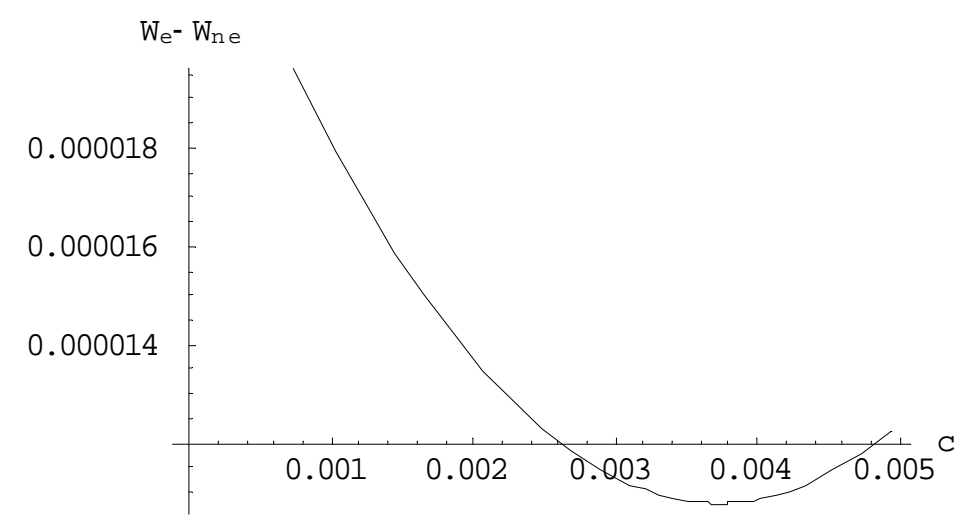

Figure 2: Subtracting (17) from (13) for $a=1, n=1, m=100$ and $c^{\max }=\frac{101}{20401}$. 


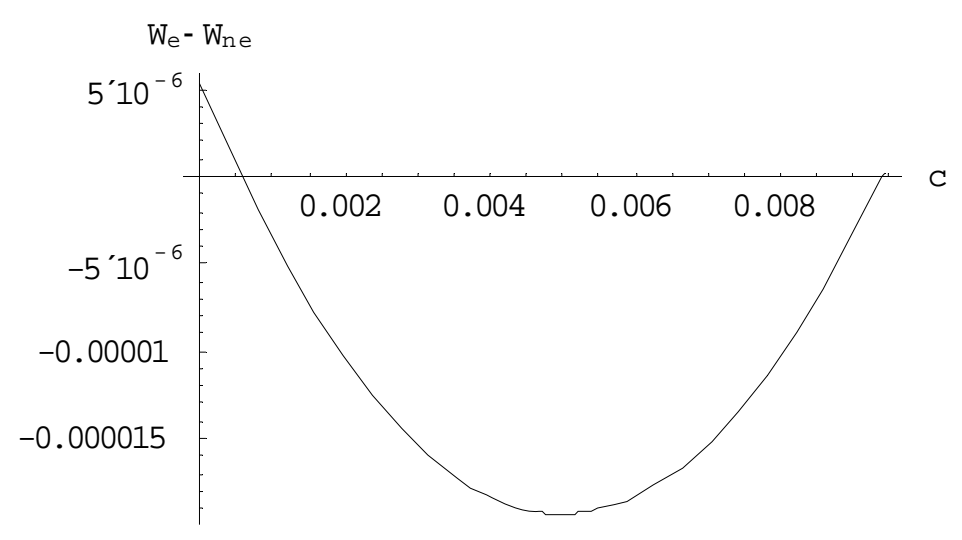

Figure 3: Subtracting (17) from (13) for $a=1, n=20, m=100$ and $c^{\max }=\frac{2020}{214220}$.

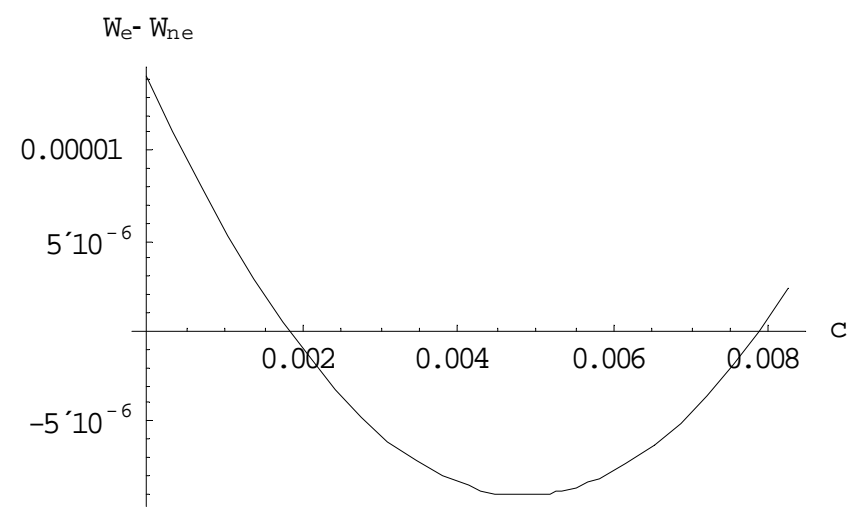

Figure 4: Subtracting (17) from (13) for $a=1, n=5, m=100$ and $c^{\max }=\frac{505}{61205}$. 
